Yervant Terzian, Daniel Weedman, Edward Khachikian, eds.

\title{
Strong Radio Outbursts in Six Active Galactic Nuclei in 1997-1998
}

\author{
Y.Y. Kovalev \\ Astro Space Center of the Lebedev Physical Institute, \\ Profsoyuznaya 84/32, Moscow, 117810 Russia
}

\begin{abstract}
The beginnings of strong radio flares in six AGNs were detected and their evolution was studied using $1-22 \mathrm{GHz}$ five epoch instantaneous spectral observations in 1997-1998. The phenomena started at the highest frequency and moved to the lower frequency in a regular fashion. Such behavior of the flares in AGNs can be explained by synchrotron emission from a compact relativistic jet implying different physical models which are discussed. For different types of AGNs the same behavior of flares is revealed, in favor of the same basic physical model for BL Lacs and quasars. A birth of new VLBI components in compact jets associated with these outbursts is predicted.
\end{abstract}

\section{Observations}

Observations were made at $31,13,7.6,3.9,2.7$, and $1.4 \mathrm{~cm}$, simultaneously for all frequencies, with the RATAN-600 in the framework of a monitoring program (see Kovalev, 1998). The beginnings of strong radio flares in some AGNs were detected in 1997. We selected six of them for an analysis: quasars $0906+01$ $(z=1.02), 1622-25(z=0.79), 1958-17(z=0.65), 2121+05(z=1.94)$, BL Lacertae object $0235+16(z=0.94)$, quasar or compact galaxy $0007+10(z=0.09)$.

\section{Radio flares evolution}

Comparing the behavior of flares with the modeled synchrotron radiation of a blob of relativistic particles evolving in a radial magnetic field (see e.g. Kovalev \& Mikhailutsa, 1980; Kovalev \& Larionov, 1994), one can see a very good agreement between them. In this model the outbursts and their evolution are explained by an increasing and a decreasing of the density of relativistic particles, emitted from the core region to the jet.

Considering the flare evolution in respect to the model of a shock wave passing through a conical adiabatically expanding jet (Marsher \& Gear, 1985), we have analyzed the different stages of flares. During a growth the synchrotron self-absorption turnover is going to lower frequencies and the turnover flux is increasing (a Compton phase). During a plateau phase the turnover frequency decreases while the turnover flux remains approximately constant (a synchrotron phase). A fading of a flare is well consistent with a final adiabatic expansion phase, both the turnover frequency and the flux decreasing in time. 
The similar behavior of flares is revealed for all objects, which are displayed on figure 1 (note that the strength of flares and the velocities of evolution are different). This favors the hypothesis of the same basic physical model for BL Lacs and quasars. The change of the polarization of a VLBI core at the beginning of these flares could happen (as was mentioned for BL Lacs by Gabuzda et al., 1994). We predict a birth of new VLBI components in compact jets associated with these outbursts. These components may appear in the VLBI maps in a few years (depending on the source) after the beginning of a flare, if a compact jet will be resolved from a VLBI core.
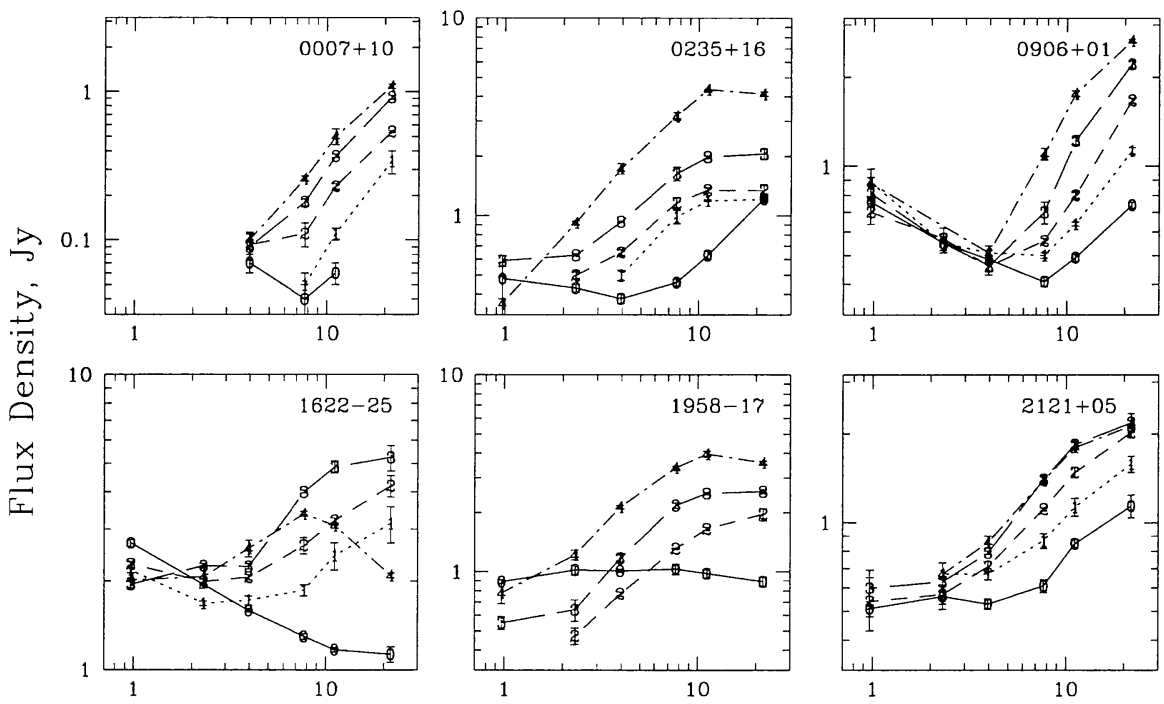

Frequency, GHz

Figure 1. The evolution of the instantaneous total flux density spectra for six AGNs. '0', '1', '2', '3', '4' denote points at March, June, September, December, 1997, and April, 1998, respectively.

Acknowledgments. The author is thankful to the LOC of the IAU Symposium 194 and INTAS for support of his stay in Byurakan during the conference and to the ISSEP for support by the PhD student grant. This research was supported in part by the Russian State Program "Astronomy".

\section{References}

Gabuzda, D.C., et al. 1994, ApJ, 435, 140

Kovalev, Yu.A., \& Mikhailutsa, V.P. 1980, Soviet Ast., 24, 400

Kovalev, Yu.A. 1998, Bull. SAO, 44, 50

Kovalev, Y.Y., \& Larionov, G.M. 1994, ALett., 20, 3

Marsher, A.P., \& Gear, W.K. 1985, ApJ, 298, 114 